\title{
СТРУКТУРА РЕГИСТРОВ ЭНДОКРИННЫХ ЗАБОЛЕВАНИЙ
}

\author{
Мокрышева Н.Г., Шестакова М.В., Мельниченко Г.А., Петеркова В.А., Викулова О.К., Рожинская Л.Я., Белая Ж.Е., \\ Дзеранова Л.К., Пигарова Е.А., Айнетдинова А.Р., Железнякова А.В., Ковалева Е.В, Еремкина А.К., Луценко А.С., \\ Вадина Т.А., Серков А.А., Дедов И.И.
}

ФГБУ «НМИЦ эндокринологии» Минздрава России, Москва

ЦЕЛЬ: с целью клинико-эпидемиологического мониторинга эндокринных заболеваний в Российской Федерации под эгидой ФГБУ «НМИЦ эндокринологии» Минздрава России была создана информационноаналитическая платформа с онлайн-форматом ввода данных, включающая 4 регистра эндокринопатий.

МАТЕРИАЛЫ И МЕТОДЫ: объекты исследования: Федеральный регистр сахарного диабета (СД) (http://diaregistry.ru); Регистр опухолей гипоталамо-гипофизарной области (ОГГО) (http://oggo.clin-reg.ru/), Регистр первичного гиперпаратиреоза (ПГПТ) (http://pgpt.clin-reg.ru/), Регистр гипопаратиреоза (гипоПТ) (http://gipopt.clin-reg.ru/). Проведена оценка баз данных на 01.06.2021 г.

РЕзУЛЬТАТЫ: Федеральный регистр СД содержит сведения о 4873155 пациентах (31\% мужчин, $\mathrm{n}=150678$ и 69\% женщин, $\mathrm{n}=3362$ 477) из 84 регионов РФ, из них: СД 1 типа - 5,5\%, $\mathrm{n}=267$ 800, СД 2 типа $92,4 \%, n=4502$ 840, другие типы СД - 2,1\%, n=102 515. Средний возраст пациентов составил 65 лет.

Регистр ОГГО содержит сведения о 10394 (26\% мужчин, n=2702 и 74\% женщин, n=7692) пациентах из 65 регионов РФ, из них: акромегалия - 45,5\%, $\mathrm{n}=4726$, пролактинома $-23,9 \%, \mathrm{n}=2488$, болезнь Иценко-Кушинга - 8,5\%, $\mathrm{n}=888$, неактивная опухоль гипофиза - 11,2\%, $\mathrm{n}=1167$, другие опухоли - 4,0\%, $\mathrm{n}=418$, редкие аденомы гипофиза $-1,6 \%, \mathrm{n}=170$. Средний возраст пациентов в регистре - 56 лет.

Регистр ПГПТ содержит сведения о 4327 пациентах (8\% мужчин, $\mathrm{n}=346$ и 92\% женщин, $\mathrm{n}=3981$ ) из 79 регионов РФ, из них: пациенты с активной фазой заболевания $-59,0 \%, \mathrm{n}=2552$, в фазе ремиссии - 33,4\%, $\mathrm{n}=1445$, рецидив у 4,9\%, $\mathrm{n}=214$, нет данных у 2,7\%, $\mathrm{n}=116$. Средний возраст пациентов составил 64 года.

Регистр гипоПТ содержит сведения о 522 пациентах (13\% мужчин, $\mathrm{n}=68$ и 87\% женщин, $\mathrm{n}=454$ ) из 63 регионов РФ, из них: послеоперационный гипоПТ - 88,9\%, $\mathrm{n}=464$, аутоиммунный гипоПТ в составе аутоиммунного полиэндокринного синдрома (АПС) $-2,5 \%, \mathrm{n}=13$, наследственный гипоПТ (кроме АПС) - 1,3\%, $\mathrm{n}=7$, идиопатический гипоПт - 6,7\%, n=35, другое - 0,6\%, n=7. Средний возраст пациентов составил 51 год.

ВЫводы: данные регистров позволяют проводить оценку эпидемиологических характеристик, мониторинг клинических данных, анализ ведения и исходов лечения пациентов с эндокринными заболеваниями, что является научно-практической основой для совершенствования качества оказания медицинской помощи.

КЛЮЧЕВЫЕ СЛОВА: регистры; сахарный диабет; опухоли гипоталамо-гипофизарной области; первичный гиперпаратиреоз; гunonapamupeos. 